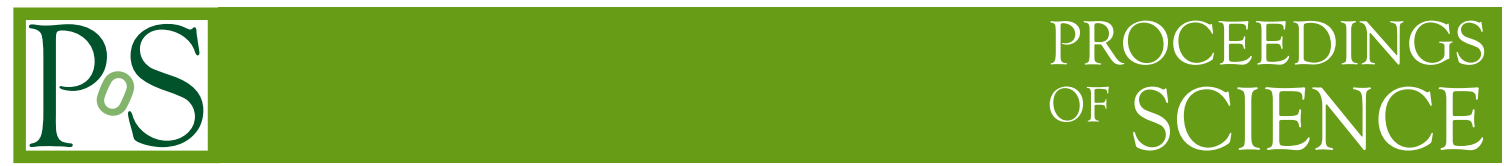

\title{
Leptogenesis via varying Weinberg operator
}

\author{
Silvia Pascoli \\ IPPP, Department of Physics, Durham University, Durham DH1 3LE, United Kingdom \\ E-mail: silvia.pascoliedurham.ac.uk
}

\section{Jessica Turner}

Theoretical Physics Department, Fermi National Accelerator Laboratory, P.O. Box 500,

Batavia, IL 60510, USA

E-mail: jessica.turner@durham.ac.uk

\section{Ye-Ling Zhou*}

IPPP, Department of Physics, Durham University, Durham DH1 3LE, United Kingdom

E-mail: ye-ling. zhouddurham.ac.uk

The Weinberg operator is a well-known solution to explain light neutrino masses. This operator violates lepton number and can provide non-equilibrium dynamics in the early Universe due to the suppression of tiny neutrino masses. A lot of underlying symmetries proposed in the lepton sector strongly motivate the existence of phase transitions at high scale. During the phase transition, the coupling of the Weinberg operator may be time-dependent, and the lepton asymmetry is generated by the interference of Weinberg operators at different times. This mechanism does not require any explicit new particles beyond the standard model or seesaw models.

EPS-HEP 2017, European Physical Society conference on High Energy Physics 5-12 July 2017

Venice, Italy

${ }^{*}$ Speaker. 


\section{Introduction}

Neutrino oscillations combined with other observations have confirmed that neutrinos have tiny masses [1]. Without extending the particle content of the Standard Model (SM), the only method of generating neutrino masses is from higher-dimensional operators. And the simplest one is the Weinberg operator [2], a dimension-5 operator violating the lepton number,

$$
\mathscr{L}_{\mathrm{W}}=\frac{\lambda_{\alpha \beta}}{\Lambda} \ell_{\alpha L} H C \ell_{\beta L} H+\text { h.c. }
$$

In order to realise sub-eV scale neutrino masses, the UV-complete scale $\Lambda$ should be around $10^{15}$ $\mathrm{GeV}$ if the coefficient $\lambda \sim \mathscr{O}(1)$. The generated neutrino masses are Majorana masses. There are several ways of UV completing this operator and they are dependent on the new particle spectra. The simplest model is the type-I seesaw, where only right-handed (RH) neutrinos are introduced.

Theories proposed for the origin of neutrino masses, on the other hand, provide an important scheme to understand the matter-antimatter asymmetry in the Universe. It is a mystery that our visible Universe is mostly formed by baryon, not antibaryon. The abundance of baryons [1]

$$
5.8 \times 10^{-10}<\eta_{B} \equiv \frac{n_{B}}{n_{\gamma}}<6.6 \times 10^{-10}(95 \% \mathrm{CL}) .
$$

One of the most widely studied mechanisms for explaining this asymmetry is baryogenesis via leptogenesis, first suggested by Fukugita and Yanagida [3]. This theory proposes that a net lepton asymmetry is first produced above the EW scale and then converted into a baryon asymmetry through the $(B-L)$-preserving weak sphaleron processes [4]. In most mechanisms of leptogenesis, RH neutrinos, as well as the seesaw framework, play a key role to generate the lepton asymmetry. In this paper, we will introduce a totally different mechanism of leptogenesis, where only the Weinberg operator and a CP-violating phase transition are required. The mechanism is insensitive to any explicit new particles in the UV-complete scale of the Weinberg operator.

\section{The mechanism}

For a successful baryogenesis, Sakharov's three conditions must be satisfied: baryon number violation, $\mathrm{C} / \mathrm{CP}$ violation and a departure from thermal equilibrium [5]. In mechanisms of baryogenesis via leptogenesis, these conditions can be simply tuned by replacing the baryon number violation by the SM lepton number and $B-L$ violation, and without doubt, all conditions have to be satisfied before the decouple of sphaleron processes. The details of dynamics that generate the lepton asymmetry depend on how these conditions are satisfied. In the original well-known thermal leptogenesis, the decay of the lightest RH neutrino $N_{1}$ provides out-of-equilibrium dynamics, and the CP violation between $N_{1} \rightarrow \ell H$ and $N_{1} \rightarrow \bar{\ell} H^{*}$ generates a net lepton asymmetry. Another mechanism of leptogenesis comes from the oscillations of RN neutrinos [6]. This mechanism assumes that the RH neutrinos have very small Yukawa couplings with the active neutrinos, $Y_{v} \sim 10^{-7}$. Thus, the RH neutrinos, produced from the Yukawa couplings, have not reached thermal equilibrium before the decouple of sphaleron processes. An asymmetry between RH neutrino chiralities is generated via oscillation during $\mathrm{RH}$ neutrino propagation and converted to the SM 
lepton-antilepton asymmetry via the Yukawa couplings. In both mechanisms, seesaw models and RH neutrinos play an essential role.

We proposed in [7] that the Sakharov conditions can be satisfied in a totally different way. Instead of including any seesaw models, we directly focus on the Weinberg operator:

- The Weinberg operator violates the lepton number and $B-L$. In the early Universe, with a thermal background, the Weinberg operator leads to the following lepton-number-violating (LNV) processes:

$$
H^{*} H^{*} \leftrightarrow \ell \ell, \quad \bar{\ell} H^{*} \leftrightarrow \ell H, \quad \bar{\ell} H^{*} H^{*} \leftrightarrow \ell, \quad \bar{\ell} \leftrightarrow \ell H H, \quad H^{*} \leftrightarrow \ell \ell H, \quad 0 \leftrightarrow \ell \ell H H .
$$

- As originally proposed for explaining tiny neutrino masses, the Weinberg operator is highly suppressed. The rate of the LNV processes induced by the Weinberg operator is approximately $\Gamma_{\mathrm{W}} \sim \frac{1}{4 \pi} m_{v}^{2} T^{3} / v_{H}^{4}$, where the Higgs VEV $v_{H}=246 \mathrm{GeV}$. For temperature $T<10^{12}$ $\mathrm{GeV}, \Gamma_{\mathrm{W}}$ is already smaller than the Hubble expansion rate $H_{u} \sim 10 T^{2} / M_{\mathrm{pl}}$. Therefore, the Weinberg operator can directly provide out-of-equilibrium dynamics in the early Universe.

- In order to generate a $\mathrm{CP}$ violation, we assume a $\mathrm{CP}$-violating phase transition which triggers a time-varying Weinberg operator. The phase transition is strongly motivated by the plenty of underlying symmetry proposed in the lepton sector, e.g., $U(1)_{B-L}$ symmetry and variety of flavour symmetries. During the phase transition, the coefficient of the Weinberg operator is time-dependent $\lambda(t)$, and a CP violation can be generated by the interference of Weinberg operators at different times.

Furthermore, as the Weinberg operator is not in equilibrium, it cannot provide enough washout for any generated lepton asymmetry. Therefore, we can safely ignore the washout effect if there is no additional LNV source beyond the Weinberg operator.

\section{Lepton asymmetry}

We calculate the lepton asymmetry in the closed-time-path (CTP) formalism. The advantage for applying this approach to our mechanism is that instead of deriving the CP-violating condition for each processes listed in Eq. (2.1), we calculate the lepton doublet self energy $\Sigma^{<,>}$as shown in Fig. 1.

We first give a brief description of the CTP approach. For the Higgs $H$ and lepton doublets $\ell_{\alpha}$ (for $\alpha=e, \mu, \tau$ ) in the CTP formalism, one defines the following Wightman propagators

$$
\begin{array}{lll}
\Delta^{<,>}\left(t_{1}, t_{2} ; \vec{x}_{1}, \vec{x}_{2}\right) & =\left\langle H^{*}\left(t_{2}, \vec{x}_{2}\right) H\left(t_{1}, \vec{x}_{1}\right)\right\rangle, & \left\langle H\left(t_{1}, \vec{x}_{1}\right) H^{*}\left(t_{2}, \vec{x}_{2}\right)\right\rangle, \\
S_{\alpha \beta}^{<,>}\left(t_{1}, t_{2} ; \vec{x}_{1}, \vec{x}_{2}\right) & =-\left\langle\bar{\ell}_{\beta}\left(t_{2}, \vec{x}_{2}\right) \ell_{\alpha}\left(t_{1}, \vec{x}_{1}\right)\right\rangle, & \left\langle\ell_{\alpha}\left(t_{1}, \vec{x}_{1}\right) \bar{\ell}_{\beta}\left(t_{2}, \vec{x}_{2}\right)\right\rangle,
\end{array}
$$

respectively, where the fermion spinor and electroweak gauge indices have been suppressed. The asymmetry between lepton and antilepton momentum distributions $L_{\vec{k}} \equiv f_{l \vec{k}}-f_{\overrightarrow{l k}}$ can be directly defined via the Wightman propagators as $L_{\vec{k}}=-\int_{t_{i}}^{t_{f}} d t_{1} \partial_{t_{1}} \operatorname{tr}\left[\gamma_{0} S_{\vec{k}}^{<}\left(t_{1}, t_{1}\right)+\gamma_{0} S_{\vec{k}}^{>}\left(t_{1}, t_{1}\right)\right]$. Ignoring the washout and using the equations of motion of $S^{<,>}$, the asymmetry is explicitly written as

$$
L_{\vec{k}}=-\int_{t_{i}}^{t_{f}} d t_{1} \int_{t_{i}}^{t_{f}} d t_{2} \operatorname{tr}\left[\Sigma_{\vec{k}}^{>}\left(t_{1}, t_{2}\right) S_{\vec{k}}^{<}\left(t_{2}, t_{1}\right)-\Sigma_{\vec{k}}^{<}\left(t_{1}, t_{2}\right) S_{\vec{k}}^{>}\left(t_{2}, t_{1}\right)\right] .
$$




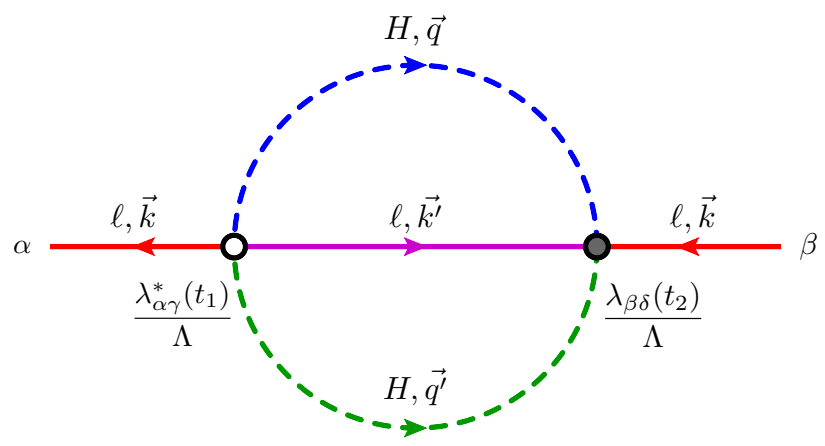

Figure 1: Lepton doublet self-energy correlation induced by the time-dependent Weinberg operator.

The self energy $\Sigma_{\vec{k}}^{<,>}\left(x_{1}, x_{2}\right)$ contributed from the Weinberg operator has been shown in Fig. 1 and is analytically written as

$$
\Sigma_{\alpha \beta, \vec{k}}^{<,>}\left(t_{1}, t_{2}\right)=\frac{12}{\Lambda^{2}} \sum_{\gamma \delta} \lambda_{\alpha \gamma}^{*}\left(t_{1}\right) \lambda_{\delta \beta}\left(t_{2}\right) S_{\gamma \delta}^{>,<}\left(t_{2}, t_{1}\right) \Delta^{>,<}\left(t_{2}, t_{1}\right) \Delta^{>,<}\left(t_{2}, t_{1}\right) .
$$

The quantitative result of the lepton asymmetry depends on both the thermal properties of the Higgs and leptons and the time-dependent behaviour of $\lambda(t)$ during the phase transition. We including their effects as follows.

The lepton doublets participate in the SM electroweak gauge interactions, and the Higgs partakes in both the electroweak gauge interaction and the top Yukawa coupling. These interactions result in thermal damping effects. These contributions are effectively included as the thermal widths $\left(\gamma_{\ell}, \gamma_{H}\right)$ to the propagators for lepton doublets and the Higgs, respectively. In the massless limit, the re-summed lepton and Higgs propagators are effective written as

$$
\begin{aligned}
& S_{\vec{k}}^{<,>}\left(t_{1}, t_{2}\right)=-P_{L} \frac{\gamma^{0} \cos \left(k y^{\mp}\right)+i \vec{\gamma} \cdot \hat{\vec{k}} \sin \left(k y^{\mp}\right)}{2 \cosh (k \beta / 2)} e^{-\gamma_{\ell, \vec{k}}|y|}, \\
& \Delta_{\vec{q}}^{<,>}\left(t_{1}, t_{2}\right)=\frac{\cos \left(q y^{\mp}\right)}{2 q \sinh (q \beta / 2)} e^{-\gamma_{H, \vec{q}}|y|},
\end{aligned}
$$

in momentum space, where $k=|\vec{k}|, q=|\vec{q}|, y^{\mp}=y \mp i \beta / 2$, and $\hat{k}=\vec{k} / k$ [8]. Additionally, for temperature above the EW scale, the other underlying sizeable couplings beyond the SM may also contribute to the propagators of leptons and the Higgs and modify the values of $\gamma_{\ell}$ and $\gamma_{H}$.

The behaviour of the time-dependent coefficient $\lambda(t)$ modifies the lepton asymmetry quantitatively. We assume a single-scalar phase transition for simplicity. Thus, $\lambda(t)$ is expressed as

$$
\lambda_{\alpha \beta}(t)=\lambda_{\alpha \beta}^{0}+\lambda_{\alpha \beta}^{1}\langle\phi(t)\rangle / v_{\phi} .
$$

During the vacuum phase transition from the trivial phase $\langle\phi\rangle=0$ to the phase $\langle\phi\rangle=v_{\phi}$, the coupling $\lambda(t)$ at a fixed spatial point ${ }^{1}$ time-dependently varies from the initial value $\lambda^{0}$ to the final value $\lambda \equiv \lambda^{0}+\lambda^{1}$. Note that the origin of the initial value $\lambda^{0}$ is not essential for our discussion. It

\footnotetext{
${ }^{1}$ The Weinberg operator may be also spatial-dependent. This part has no contribution to the lepton asymmetry and will not be considered here [7].
} 
could be resulted from another scalar acquiring a VEV before $\phi$ gets the VEV $v_{\phi}$, or independent of the phase transition. This assumption allows us to integrate out the time $t_{1}$ and $t_{2}$ analytically.

After the simplification of the phase space, we obtain the final lepton asymmetry as

$$
L_{\vec{k}}=\frac{3 \operatorname{Im}\left\{\operatorname{tr}\left[m_{v}^{0 *} m_{v}\right]\right\} T^{2}}{(2 \pi)^{4} v_{H}^{4}} F\left(\frac{k}{2 T}, \frac{\gamma}{T}\right)
$$

where $m_{v}^{0}=\lambda^{0} v_{H}^{2} / \Lambda, m_{v}=\lambda v_{H}^{2} / \Lambda, \gamma=\gamma_{\ell}+\gamma_{H}$, and $F(k / 2 T, \gamma / T)$ is a loop factor. The loop factor in general provides an $\mathscr{O}(10)$ factor enhancement [7]. Without loss of generality, one may assume $\operatorname{Im}\left\{\operatorname{tr}\left[m_{v}^{0} m_{v}^{*}\right]\right\} \sim m_{v}^{2} \sim(0.1 \mathrm{eV})^{2}$. In order to generate enough baryon asymmetry, $\Delta f_{\vec{l} \vec{k}} \sim \eta_{B}$, the temperature for the phase transition approximates to $T \sim 10^{11} \mathrm{GeV}$.

\section{Summary}

Most mechanisms of leptogenesis are limited in the seesaw framework and RH neutrinos play an essential role. We propose a novel mechanism insensitive to the details of any seesaw models. In this mechanism, the lepton asymmetry is generated directly by a varying Weinberg operator. The Weinberg operator violates the lepton number, and it is sufficiently tiny, automatically providing out-of-equilibrium dynamics in the early Universe. In order to generate a CP violation, we introduce a phase transition, which is strongly motivated by a lot of underlying symmetries, e.g., $B-L$ symmetry and flavour symmetries. During the phase transition, the Weinberg operator is time-dependent, and the lepton asymmetry is generated from the interference of Weinberg operators at different times. To generate enough baryon asymmetry, The temperature of phase transition to allow for successful baryogenesis is roughly around $10^{11} \mathrm{GeV}$.

Acknowledgement. This work was supported by the European Research Council under ERC Grant "NuMass" (FP7-IDEAS-ERC ERC-CG 617143).

\section{References}

[1] K. A. Olive et al. [Particle Data Group Collaboration], Chin. Phys. C 38 (2014) 090001.

[2] S. Weinberg, Phys. Rev. Lett. 43 (1979) 1566.

[3] M. Fukugita and T. Yanagida, Phys. Lett. B 174 (1986) 45.

[4] S. Y. Khlebnikov and M. E. Shaposhnikov, Nucl. Phys. B 308 (1988) 885.

[5] A. D. Sakharov, Pisma Zh. Eksp. Teor. Fiz. 5, 32 (1967) [JETP Lett. 5, 24 (1967)] [Sov. Phys. Usp. 34 (1991) 392] [Usp. Fiz. Nauk 161 (1991) 61].

[6] E. K. Akhmedov, V. A. Rubakov and A. Y. Smirnov, Phys. Rev. Lett. 81 (1998) 1359 [hep-ph/9803255].

[7] S. Pascoli, J. Turner and Y. L. Zhou, arXiv:1609.07969 [hep-ph].

[8] A. Anisimov, W. Buchmüller, M. Drewes and S. Mendizabal, Annals Phys. 326, 1998 (2011) Erratum: [Annals Phys. 338 (2011) 376] [arXiv:1012.5821 [hep-ph]]. 